\title{
Bioinspired Disordered Flexible Metasurfaces for Human Tear Analysis Using Broadband Surface-Enhanced Raman Scattering
}

\author{
Vinayak Narasimhan, Radwanul Hasan Siddique, Haeri Park, and Hyuck Choo*
}

Cite This: ACS Omega 2020, 5, 12915-12922

Read Online

ABSTRACT: Flexible surface-enhanced Raman scattering (SERS) has received attention as a means to move SERS-based broadband biosensing from bench to bedside. However, traditional flexible periodic nanoarrangements with sharp plasmonic resonances or their random counterparts with spatially varying uncontrollable enhancements are not reliable for practical broadband biosensing. Here, we report bioinspired quasi(dis)ordered nanostructures presenting a broadband yet tunable application-specific SERS enhancement profile. Using simple, scalable biomimetic fabrication, we create a flexible metasurface (flex-MS) of quasi(dis)ordered metal-insulator-metal (MIM) nanostructures with spectrally variable, yet spatially controlled electromagnetic hotspots. The MIM is designed to simultaneously localize the electromagnetic signal and block background Raman signals from the underlying polymeric substrate-an
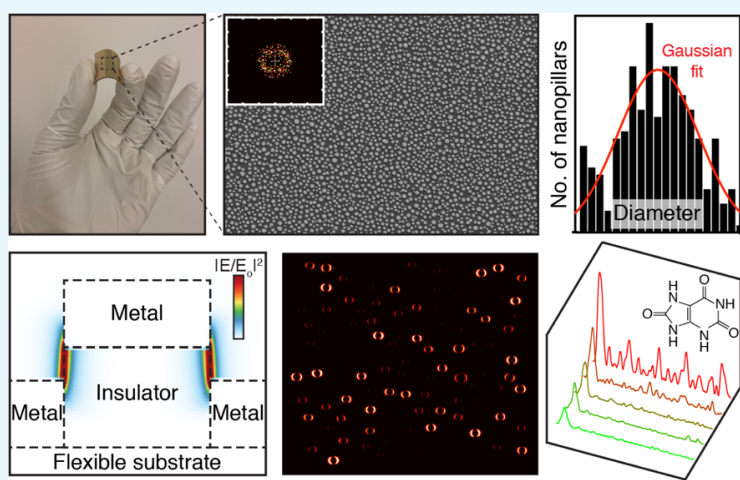

inherent problem of flexible SERS. We elucidate the effect of quasi-
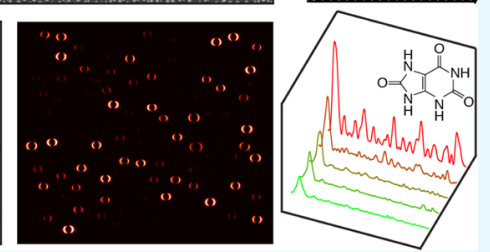
(dis)ordering on broadband tunable SERS enhancement and employ the flex-MS in a practical broadband SERS demonstration to detect human tear uric acid within its physiological concentration range $(25-150 \mu \mathrm{M})$. The performance of the flex-MS toward noninvasively detecting whole human tear uric acid levels ex vivo is in good agreement with a commercial enzyme-based assay.

\section{INTRODUCTION}

Surface-enhanced Raman scattering (SERS) has shown great promise as a technique for molecular fingerprinting because of its high sensitivity and selectivity along with its inherent simplicity. ${ }^{1-3}$ In particular, SERS from flexible substrates has received great attention recently owing to its advantages over rigid substrates. ${ }^{4-6}$ For instance, flexible SERS can be used on irregular surfaces for in situ biosensing thereby overcoming complex analyte extraction strategies and other sample preparation steps required while using conventional rigid substrates. However, the adoption of flexible SERS as a pointof-care diagnostic tool has been limited by a number of factors. ${ }^{6}$ First, most structures used for this application are designed either with periodic arrangements because of their highly predictable plasmonic resonances ${ }^{7,8}$ or with random arrangements because of their ease of fabrication. ${ }^{9,10}$ However, periodic structures with narrowband resonance profiles are not tunable for multiplexing. ${ }^{6-8}$ Multiplexed in situ SERS for instance would require a broadband and tunable plasmonic resonance profile to provide uniform enhancement of various Raman modes occurring at greatly differing vibrational energy states. $^{11-13}$ In the same vein, random structures do not guarantee repeatable SERS performance for a given bandwidth because of spatially varying enhancements that are not tunable. ${ }^{14}$ Second, flexible SERS platforms are usually made of polymers that generate a considerable Raman background signal. ${ }^{6}$ Finally, challenges in obtaining reproducible signal can also be attributed to the difficulty in homogeneously, scalable and cost effective manufacturing SERS active sites or hotspots. ${ }^{15}$ These issues necessitate the requirement for flexible SERS approaches with spatially uniform broadband, yet tunable plasmonic resonances that are scalable and reliably manufacturable.

Inspiration can be sought from nature which boasts a plethora of biophotonic nanostructures possessing quasi(dis)order or controlled disorder where both structural dimensions and periodicity follow unique distributions. ${ }^{16,17}$ Such an amalgamation of short-range order (i.e., periodicity) with long-range disorder (i.e., randomization) along with variations in the structure size leads to a host of useful omnidirectional broadband, yet tunable optical properties. $^{18-20}$ In this work, using a simple biomimetic fabrication process, we realize a highly scalable and flexible plasmonic metasurface-based (flex-MS) SERS platform. The flex-MS consists of a dense quasi-(dis)ordered ensemble of gold ( $\mathrm{Au}$ ) nanodisks on nanoholes separated by a sub-10 nm silicon

Received: February 14, 2020

Accepted: May 6, 2020

Published: May 18, 2020

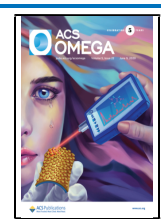


dioxide $\left(\mathrm{SiO}_{2}\right)$ nanogap in a metal-insulator-metal (MIM) configuration. The entire configuration is created at a waferscale over a $400 \mu \mathrm{m}$-thick silicone elastomer (PDMS) layer. The MIM is designed not only for light confinement and field enhancement, but is also responsible for blocking the Raman background signal from the underlying PDMS. The fabrication approach which is spin-coating based allows for a short-rangeordered Gaussian distribution of MIM nanostructure diameters resulting in a broadband, yet tunable plasmonic resonance and corresponding $\left|E / E_{0}\right|^{2}$ enhancement factor (EF). Through rigorous numerical simulations and comparative experimentation, we isolate the contributions of the bioinspired short-range periodicity (i.e., positional disorder) and the diameter distribution (i.e., size disorder) toward SERS EF. As a practical broadband SERS demonstration of the flex-MS, we perform the label-free detection of human tear uric acid (UA) toward the diagnosis various chronic pathologies. Our platform effectively tracks prominent peaks of UA at large Raman shifts within its physiological concentration range $(25-150 \mu \mathrm{M})$ in human tears. Finally, we show that the performance of the flexMS in detecting UA levels in whole human tear samples from different subjects is in good agreement with a commercial enzyme-based assay.

\section{RESULTS AND DISCUSSION}

2.1. Flex-MS Fabrication and Characterization. The flex-MS was fabricated using a simple process consisting of three steps, as shown in Figure 1a. On spin-coated PDMS thin films, the first step involves a biomimetic technique that relies on nanostructuring through the lateral phase-separation of two synthetic polymers-polystyrene (PS) and polymethyl methacrylate (PMMA) co-dissolved in methyl ethyl ketone (MEK). ${ }^{20,21}$ This approach is analogous to the formation of quasi-(dis)ordered biophotonic nanostructures on the wings

\section{a}
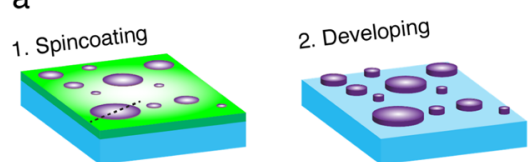

3. Evaporation
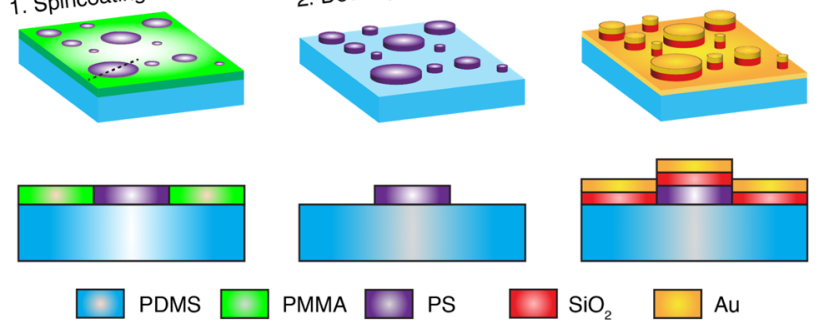

b

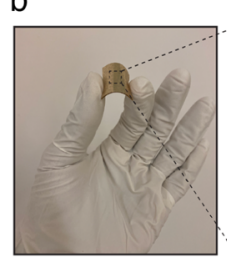

C
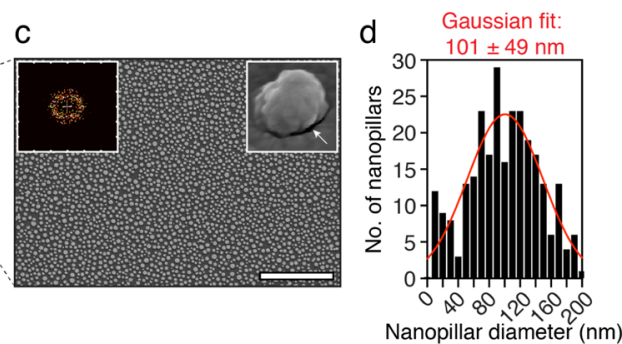

Figure 1. (a) Simple and scalable three-step fabrication process of the flex-MS. (b) Fabricated flex-MS sample with (c) quasi-(dis)ordered MIM nanostructures. Insets correspond to the 2D FFT taken to determine a short-range periodicity of $318 \pm 45 \mathrm{~nm}$ (left) and a single MIM nanostructure of diameter $100 \mathrm{~nm}$ with the sub-10 nm insulator nanogap indicated by the white arrow (right). Scale bar: $2 \mu \mathrm{m}$. (d) MIM structure diameter distribution with a Gaussian mean and SD of $101 \pm 49 \mathrm{~nm}$ and scales of birds and insects. ${ }^{22}$ The lateral phase separation of PS and PMMA occurs under spin-coating which results in densely packed quasi-(dis)ordered distribution of circular hydrophobic PS islands in a matrix of hydrophilic PMMA (Figure S1). Furthermore, through the control of various parameters such as the spin-speeds, relative humidities, polymer weight ratios, and molecular weights, the average diameter and short-range periodicity of the PS islands can be effectively tuned. ${ }^{23,24}$ Next, through selective dissolution of PMMA in acetic acid which leaves behind a nanopillar mask of PS, $\mathrm{SiO}_{2}$ is directionally deposited through E-beam evaporation. Finally, $\mathrm{Au}$ of an appropriate thickness is directionally evaporated over the $\mathrm{SiO}_{2}$ nanopillars to create a scalable flexMS with uniform sub-10 nm thick MIM nanogaps. A photograph and SEM image of the flex-MS are shown in Figure 1b,c. A 2D fast Fourier transform (FFT) of the top view SEM image as shown in the inset of Figure 1c reveals a ringshape distribution in the spatial domain that is characteristic of quasi-(dis)order in nature. ${ }^{16,17,20}$ The average short-range isotropic periodicity in this case is $318 \pm 45 \mathrm{~nm}$. Moreover, the second inset in Figure 1c shows a high-magnification SEM image of a single MIM structure confirming the sub-10 nm hotspot. Additionally, we have verified the existence of the sub$10 \mathrm{~nm}$ gap in our prior work. ${ }^{24}$ Figure $1 \mathrm{~d}$ shows a distribution of MIM structure diameters which can be estimated by a Gaussian profile fit with a mean and standard deviation (SD) of $101 \pm 49 \mathrm{~nm}$.

2.2. Single MIM Structure Simulations. The aforementioned dimensions were chosen through rigorous finitedifference time-domain (FDTD) simulations. First, as shown in Figure 2a, a single MIM structure with a $5 \mathrm{~nm}$ insulator gap was simulated to obtain the extinction profile and electric-field distribution. The plasmonic behaviors of the two metal layers (Au nanohole and Au nanodisk) couple with each other leading to strong electric-field confinement at the MIM junction. ${ }^{24}$ This effect has been demonstrated for different MIM combinations toward broadband SERS applications. ${ }^{25,26}$ Additionally, we have previously demonstrated the use of similar MIM structures with higher-order plasmonic gap modes toward plasmon-enhanced fluorescent detection of nucleic acids and suppression of fluorescence quenching. ${ }^{24} \mathrm{~A}$ modal analysis reveals that in the vis-NIR regime, this coupling results in the formation of a dipolar mode polarized along the MIM junction (Figure $2 \mathrm{~b}$ ). This, in turn, produces a very tunable and large extinction cross section and a corresponding localized $|E|^{2}$ enhancement. Figure $2 \mathrm{c}$ presents the maximum normalized $\left|E / E_{\mathrm{o}}\right|^{2}$ enhancement numerically obtained for single MIM structures ranging from 60 to $110 \mathrm{~nm}$ in diameter with a $5 \mathrm{~nm}$ gap. However, like most ordered plasmonic structures used for SERS, this mode is fairly narrowband.

2.3. Flex-MS Ensemble Simulations. To quantify the impact of quasi-(dis)ordering on the $\left|E / E_{\mathrm{o}}\right|^{2}$ enhancement, we generated an ensemble of quasi-(dis)ordered MIM structures to simulate their optical properties. Two aspects were studied: (1) short-range periodicity (i.e., positional disorder) and (2) diameter distribution (i.e., size disorder). Here, the diameters of the structures were made to obey a Gaussian distribution as with the flex-MS (diameter: $98 \pm 30 \mathrm{~nm}$ ) with short-range periodicity $(319 \pm 37 \mathrm{~nm}$ ) (Figure S2). To isolate the individual contributions of (1) and (2), periodic structures (diameter: $100 \mathrm{~nm}$, periodicity: $320 \mathrm{~nm}$ ) and short-rangeordered structures of the same diameter (diameter: $100 \mathrm{~nm}$ ) 


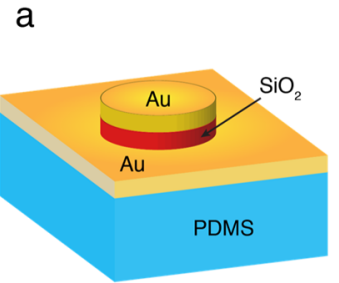

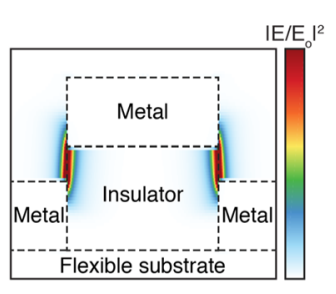

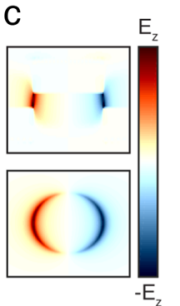

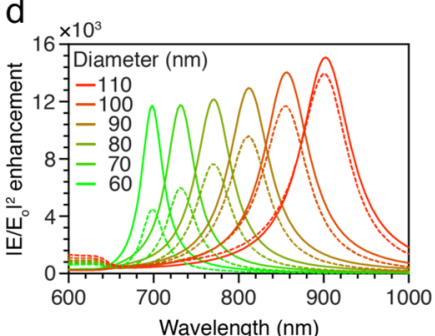

Figure 2. (a) Schematic of a single MIM nanostructure on the flex-MS platform (b) Coupling of the Au nanodisk and Au nanohole across the $\mathrm{SiO}_{2}$ nanogap results in greatly enhanced electric-field environments. (c) Field profile of the nanogap shows the presence of a vertically polarized dipolar mode. (d) Normalized maximum theoretical $\left|E / E_{\mathrm{o}}\right|^{2}$ enhancement (solid curves) and extinction cross section (dotted curves) of a single MIM nanostructure of varying diameter between 60 and $110 \mathrm{~nm}$ with a fixed gap size of $5 \mathrm{~nm}$.
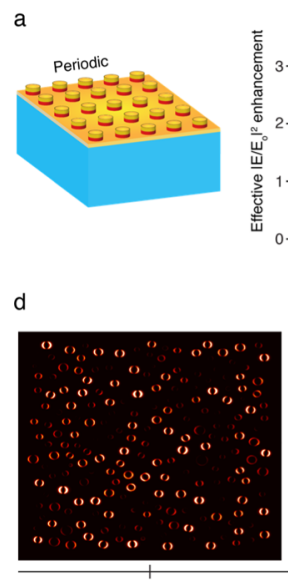
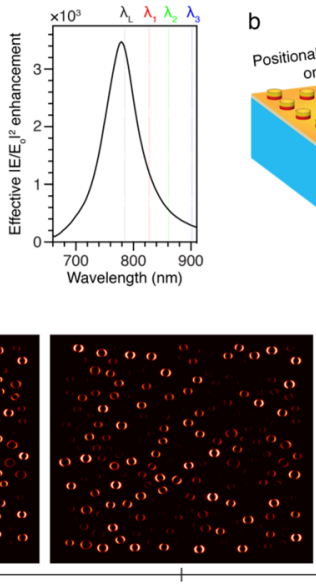

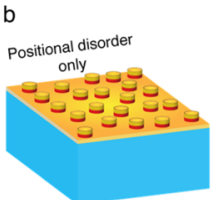

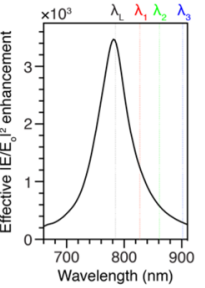

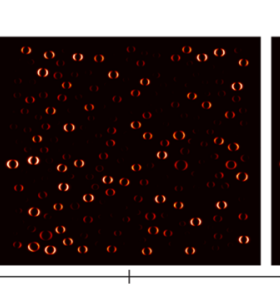

$\lambda_{2}: 861 \mathrm{~nm}$
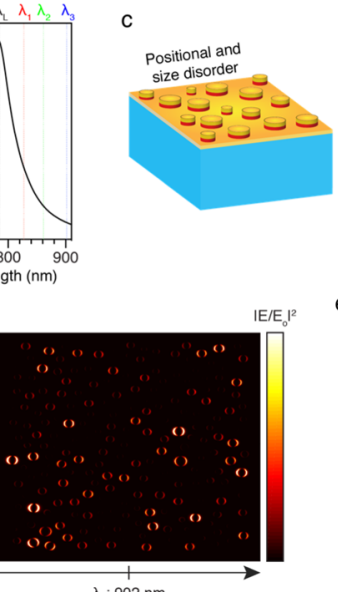

$\lambda_{3}: 902 \mathrm{~nm}$

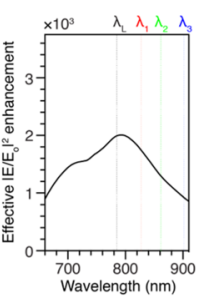

e

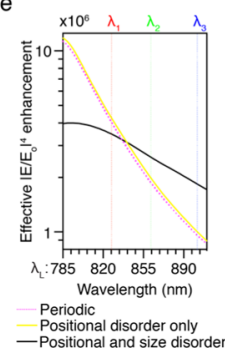

Figure 3. Ensemble of (a) periodic MIM nanostructures and (b) those with pure positional disorder demonstrating an identical narrowband effective normalized $\left|E / E_{0}\right|^{2}$ enhancement profile. (c) Effective normalized $\left|E / E_{0}\right|^{2}$ enhancement profile of a quasi-(dis)ordered MIM ensemble with both positional and size disorder is broadband compared to $(\mathrm{a}, \mathrm{b})$. The $\left|E / E_{\mathrm{o}}\right|^{2}$ enhancement profile encompasses the excitation and the Ramanshifted wavelengths $\left(\lambda_{\mathrm{L}}, \lambda_{1}, \lambda_{2}\right.$, and $\left.\lambda_{3}\right)$ of relevance in this work. (d) Field-map spanning the $3.5 \times 3.5 \mu \mathrm{m}$ array at $\lambda_{\mathrm{L}}, \lambda_{1}, \lambda_{2}$, and $\lambda_{3}$ shows the progressive excitation of first small and then larger MIM nanostructures with increasing wavelength. (e) Effective $\left|E / E_{\mathrm{o}}\right|^{4}$ enhancement from periodic structures, structures with a positional disorder only and those with both positional and size disorder. This effective enhancement was numerically computed as $\operatorname{EF}\left(\omega_{\mathrm{L}}, \omega_{\mathrm{R}}\right)$.

were considered. For the three categories of structures, an insulator gap of $9 \mathrm{~nm}$ was chosen to tune the resonance close to the laser wavelength $\left(\lambda_{\mathrm{L}}=785 \mathrm{~nm}\right.$ ) (Figure S3). The effective normalized $\left|E / E_{\mathrm{o}}\right|^{2}$ enhancement in each case (averaged over 4000 individual hotspots) was computed not only at the laser wavelength $\left(\lambda_{\mathrm{L}}=785 \mathrm{~nm}\right)$, but also the Raman-shifted wavelengths of relevance $\left(\lambda_{1}, \lambda_{2}\right.$, and $\lambda_{3}=827$, 861 , and $902 \mathrm{~nm}$ ) for this work. For periodic structures, the resonance profile is predictably governed by the collective gapplasmon resonance of individual MIM structures that dominate any weaker in-plane lattice effects (Figure 3a). ${ }^{27}$ This is evidenced by the fact that the periodic array resonance position and mode shape is identical to that of a single MIM structure (diameter: $100 \mathrm{~nm}$, gap: $9 \mathrm{~nm}$ ) (Figure S4). Next, quasi-(dis)ordered structures with the same diameter (i.e., positional disorder) were studied. The effect of the pure positional disorder retains the collective gap-plasmon resonance of individual MIM structures marked by the same resonance position and intensity as that of the periodic array given the same number of averaged hotspots (Figure $3 b$ ). In other words, for a periodicity that is large enough to ensure either weak or no coupling between adjacent nanostructures, the effect of the short-range order (i.e., pure positional disorder) is negligible. ${ }^{28,29}$ Finally, the introduction of a Gaussian distribution of diameters along with the short-range order (i.e., size and positional disorder) produces a considerably more broadband $\left|E / E_{\mathrm{o}}\right|^{2}$ enhancement compared to the periodic structures as shown in Figure $3 c$ because of the resonance of subsets of MIM structures of a given diameter (Figures 3d and S5). ${ }^{28}$ The bandwidth of the $\left|E / E_{\mathrm{o}}\right|^{2}$ enhancement of size-disordered structures $(\sim 219 \mathrm{~nm})$ was 3.4 times larger than that of the periodic structures $(\sim 65 \mathrm{~nm})$. The fractal, yet controllable nature of the MIM ensemble with a size and positional disorder produces a broadband $\left|E / E_{\mathrm{o}}\right|^{2}$ enhancement that encompasses $\lambda_{\mathrm{L}}$ and $\lambda_{1-3}$. While the periodic structures and those with a pure positional disorder provide a high effective enhancement at $\lambda_{\mathrm{L}}$ (i.e., $\left|E / E_{\mathrm{o}}\right|_{\lambda_{\mathrm{L}}}{ }^{2}$ ) of 3358 and 3430 , respectively, their enhancements at $\lambda_{1-3}$ is considerably lower (periodic: $\left|E / E_{\mathrm{o}}\right|_{\lambda_{1}}{ }^{2} \approx 1210,\left|E / E_{\mathrm{o}}\right|_{\lambda_{2}}{ }^{2} \approx 563$, and $\left|E / E_{\mathrm{o}}\right|_{\lambda_{3}}{ }^{2}$ $\approx 285$; pure positional disorder: $\left|E / E_{\mathrm{o}}\right|_{\lambda_{1}}{ }^{2} \approx 1252, \mid E / E_{\mathrm{o}} \mathrm{l}_{\lambda_{2}}{ }^{2} \approx$ 585, and $\left|E / E_{\mathrm{o}}\right|_{\lambda_{3}}^{2} \approx 292$ ). In comparison, the introduction of the size disorder provides a more uniform enhancement with I $E / E_{\mathrm{o}} \mathrm{I}_{\lambda_{\mathrm{L}}}^{2} \approx 1992,\left|E / E_{\mathrm{o}} \mathrm{I}_{\lambda_{1}}^{2} \approx 1748,\right| E / E_{\mathrm{o}} \mathrm{I}_{\lambda_{2}}{ }^{2} \approx 1305$, and $\mid E /$ $E_{\mathrm{o}} \mathrm{l}_{\lambda_{3}}^{2} \approx 921$. 

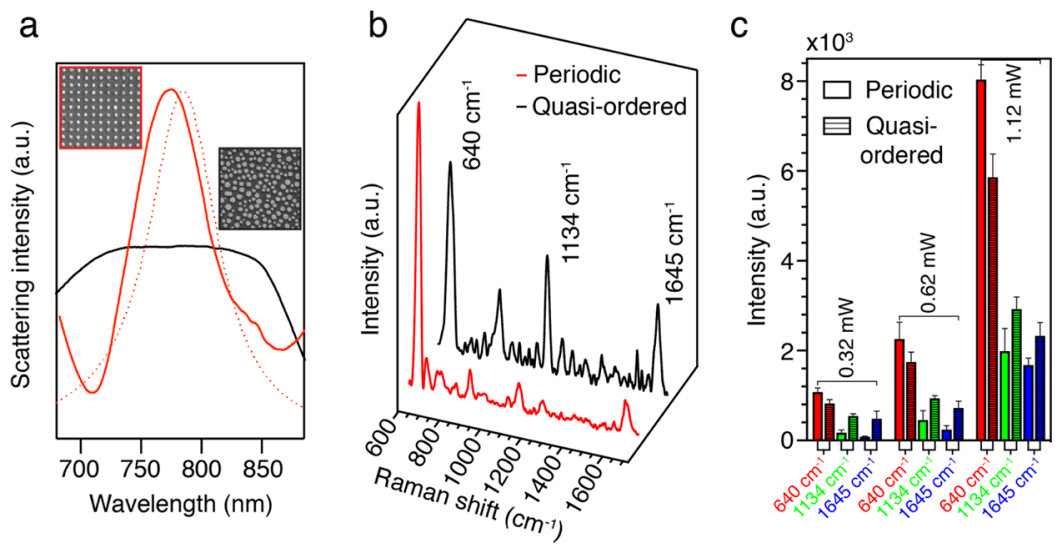

Figure 4. (a) Scattering intensity of the fabricated periodic and quasi-(dis)ordered array shown as solid lines measured using a microspectroscopic setup operating in DF mode. The simulated scattering profile of a single MIM structure (gap: $9 \mathrm{~nm}$ ) which most closely matches the experimental result is also shown as a dotted line. The SEM images of the corresponding structures are shown in the inset. (b) SERS spectra of UA measured at $0.62 \mathrm{~mW}$ laser power. While the periodic MIM array enhances the $640 \mathrm{~cm}^{-1}$ peak considerably, the flex-MS offers a more broadband enhancement of all three peaks. (c) Analysis performed for various laser powers.

2.4. Broadband SERS Enhancement Factor Computation. The origin of this broadband effect can be of great benefit to SERS. As per the frequently used $|E|^{4}$-approximation, SERS enhancement factor (EF) is typically expressed as

$$
\mathrm{EF}\left(\omega_{\mathrm{L}}, \boldsymbol{r}_{\mathrm{m}}\right)=\left|\frac{E_{\mathrm{loc}}\left(\omega_{\mathrm{L}}, \boldsymbol{r}_{\mathrm{m}}\right)}{E_{\text {inc }}\left(\omega_{\mathrm{L}}, \boldsymbol{r}_{\mathrm{m}}\right)}\right|^{4}
$$

where $E_{\mathrm{loc}}\left(\omega_{\mathrm{L}}, \boldsymbol{r}_{\mathrm{m}}\right)$ and $E_{\mathrm{inc}}\left(\omega_{\mathrm{L}}, \boldsymbol{r}_{\mathrm{m}}\right)$ are the localized and incident electric fields at a laser excitation frequency of $\omega_{\mathrm{L}}$ for a Raman dipole at position $\boldsymbol{r}_{\mathrm{m}} \cdot{ }^{30}$ Here, a significant contribution from the radiative enhancement $M_{\text {rad }}$ is overlooked in favor of electric-field enhancement $M_{\text {loc }}{ }^{31-33} M_{\text {rad }}$ measures the enhancement of the power radiated by a dipole in the presence of a plasmonic nanostructure. Factoring in this contribution, the EF can be more precisely expressed as ${ }^{30}$

$$
\begin{aligned}
\mathrm{EF}\left(\omega_{\mathrm{L}}, \omega_{\mathrm{R}}, \boldsymbol{r}_{\mathrm{m}}\right) & =M_{\mathrm{loc}}\left(\omega_{\mathrm{L}}, \boldsymbol{r}_{\mathrm{m}}\right) M_{\mathrm{rad}}\left(\omega_{\mathrm{R}}, \boldsymbol{r}_{\mathrm{m}}\right) \\
& =\left|\frac{E_{\mathrm{loc}}\left(\omega_{\mathrm{L}}, \boldsymbol{r}_{\mathrm{m}}\right)}{E_{\mathrm{inc}}\left(\omega_{\mathrm{L}}, \boldsymbol{r}_{\mathrm{m}}\right)}\right|^{2}\left|\frac{E_{\mathrm{loc}}\left(\omega_{\mathrm{R}}, \boldsymbol{r}_{\mathrm{m}}\right)}{E_{\mathrm{inc}}\left(\omega_{\mathrm{R}}, \boldsymbol{r}_{\mathrm{m}}\right)}\right|^{2}
\end{aligned}
$$

where $\omega_{\mathrm{R}}$ is the Stokes-shifted Raman scattering frequency. The $|E|^{4}$-approximation assumes that $\omega_{\mathrm{R}} \approx \omega_{\mathrm{L}}$ and as a result, $M_{\text {loc }} \approx M_{\text {rad }}$. While this approximation is accurate for $\omega_{\mathrm{L}}$ in the blue and green regime, it is inaccurate in the red and NIR regime particularly within the fingerprint region (500-1500 $\left.\mathrm{cm}^{-1}\right){ }^{32,34}$ This is because for a given vibrational mode, the difference between the vibrational and excitation energies becomes more significant for lower energy excitations. In principle, for NIR-based SERS, broadband EFs are greatly desirable as they better account for $M_{\text {rad. }}{ }^{32}$ Based on our ensemble simulations, the effective $\operatorname{EF}\left(\omega_{\mathrm{L}}, \omega_{1-3}\right)$ for the periodic structures $\left(\mathrm{EF}\left(\omega_{\mathrm{L}}, \omega_{1}\right) \approx 4.06 \times 10^{6}, \mathrm{EF}\left(\omega_{\mathrm{L}}, \omega_{2}\right) \approx\right.$ $1.89 \times 10^{6}$, and $\left.\operatorname{EF}\left(\omega_{\mathrm{L}}, \omega_{3}\right) \approx 0.96 \times 10^{6}\right)$ and those with a purely positional disorder $\left(\mathrm{EF}\left(\omega_{\mathrm{L}}, \omega_{1}\right) \approx 4.29 \times 10^{6}, \mathrm{EF}\left(\omega_{\mathrm{L}}\right.\right.$, $\left.\omega_{2}\right) \approx 2.01 \times 10^{6}$, and $\left.\operatorname{EF}\left(\omega_{\mathrm{L}}, \omega_{3}\right) \approx 1.00 \times 10^{6}\right)$ are evidently lower for $\omega_{2}$ and $\omega_{3}$ than structures with a size and positional disorder $\left(\operatorname{EF}\left(\omega_{\mathrm{L}}, \omega_{1}\right) \approx 3.48 \times 10^{6}, \operatorname{EF}\left(\omega_{\mathrm{L}}, \omega_{2}\right) \approx 2.60 \times 10^{6}\right.$, and $\operatorname{EF}\left(\omega_{\mathrm{L}}, \omega_{3}\right) \approx 1.84 \times 10^{6}$ ) (Figure $3 \mathrm{e}$ ).

The effect of the bioinspired quasi-(dis)order was then experimentally verified by comparing the optical properties of the flex-MS with periodic MIM structures (diameter: $100 \mathrm{~nm}$, periodicity: $320 \mathrm{~nm}$, gap: $9 \mathrm{~nm}$ ) fabricated via E-beam lithography. Using a microspectroscopic setup in dark-field (DF) mode, the scattering cross section of the two metasurfaces were measured in the NIR regime. This revealed the considerably more broadband scattering profile of the flexMS compared to the periodic array (Figure 4a). Furthermore, the existence of the $9 \mathrm{~nm}$ gap was verified through simulation (Figure S6). The SERS performance of the two sets of structures was compared by detecting UA-a SERS-active molecule with prominent peaks at 640,1134 , and $1645 \mathrm{~cm}^{-1}$ which originate from the skeletal ring and $\mathrm{C}-\mathrm{N}$ bond deformations. ${ }^{35}$ When excited with a $785 \mathrm{~nm}$ laser, these peaks appear at 827,861 , and $902 \mathrm{~nm}$, respectively (i.e., $\omega_{\mathrm{L}} \neq$ $\left.\omega_{\mathrm{R}}\right)$. For the same UA concentration in DI water $(150 \mu \mathrm{M})$ and three different laser powers $(0.32,0.62$, and $1.12 \mathrm{~mW})$, the three peaks under consideration were uniformly enhanced by the flex-MS compared to the periodic MIM structures (Figure $4 \mathrm{~b}, \mathrm{c}$ ). The $640 \mathrm{~cm}^{-1}$ peak was enhanced more significantly by the periodic structures compared to the flex-MS as this peak lies closest to $\omega_{\mathrm{L}}$, where the sharp plasmonic resonance of the former is tuned. However, 1134 and $1645 \mathrm{~cm}^{-1}$ peaks that were located further away from $\omega_{\mathrm{L}}$ were enhanced more significantly by the flex-MS because of its broadband enhancement profile. This property is particularly useful for sensing with low power. In our case for instance, when using a power of $0.32 \mathrm{~mW}$, the 1134 and $1645 \mathrm{~cm}^{-1}$ peaks were enhanced 3.1 and 5.7 fold by the flex-MS compared to the periodic structures. Finally, the background suppression property of the flex-MS was also verified through comparative experimentation (Figure S7).

2.5. Broadband SERS Biosensing of Tear UA. The broadband enhancement of the flex-MS platform was used to detect various concentrations of UA. Hyperuricemia (i.e., elevated levels of blood UA) has been identified as a biomarker of various diseases such as gout or gouty arthritis, ${ }^{36}$ diabetes, ${ }^{37}$ Parkinson's disease, ${ }^{38}$ renal disease, ${ }^{39}$ and cardiovascular disease, ${ }^{40}$ to name a few. While continual monitoring of UA in blood is hindered by the invasive nature of blood collection and sampling, human tears which are considerably less invasive to assay thereby proving to be an interesting alternative. ${ }^{41}$ Tears are also far less complex in constituents compared to blood and have large average concentrations of UA (68 \pm 46 


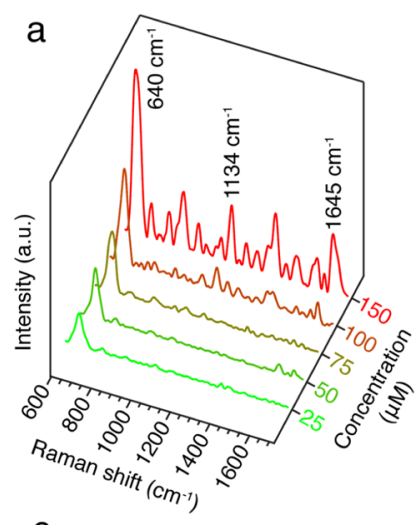

C

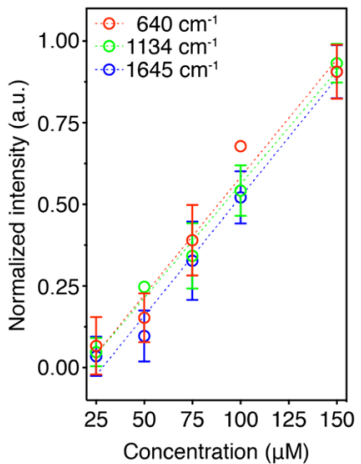

b

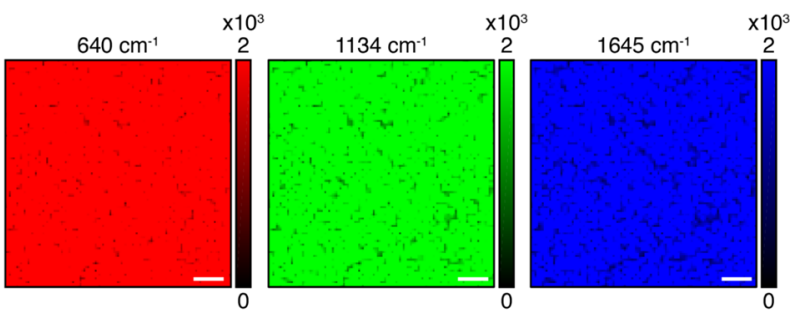

d

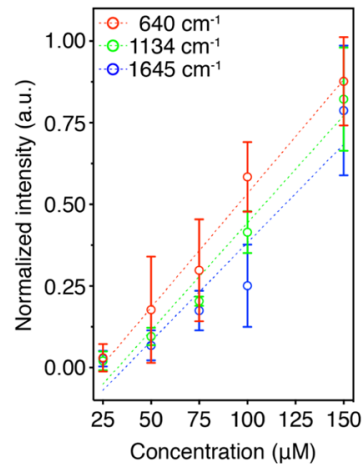

e

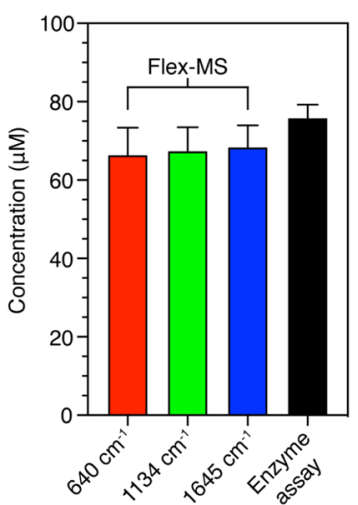

Figure 5. (a) Peaks of UA at 640,1134 , and $1645 \mathrm{~cm}^{-1}$ tracked between 25 and $150 \mu \mathrm{M}$ in PBS. (b) SERS surface mapping (UA concentration: $150 \mu \mathrm{M}$ ) over a $150 \times 150 \mu \mathrm{m}$ area at 640,1134 , and $1645 \mathrm{~cm}^{-1}$ showing spatial uniformity. Scale bars: $20 \mu \mathrm{m}$. (c) Normalized intensity of each peak shows excellent linearity. (d) 640,1134 , and $1645 \mathrm{~cm}^{-1}$ peaks tracked between 25 and $150 \mu \mathrm{M}$ in artificial tear buffer. (e) SERS performance of the flex-MS using all three peaks is compared for pooled whole tears consisting of 8 individual tear samples with a commercial enzyme-based assay. The two results are in good agreement.

$\mu \mathrm{M}) .^{10}$ Furthermore, a clear correlation between blood and tear UA levels has been established. ${ }^{10}$ As a result, assaying tear UA levels noninvasively and in a label-free fashion via flexible SERS holds great promise. Using the flex-MS, we first tracked the 640,1134 , and $1645 \mathrm{~cm}^{-1}$ peaks for UA concentrations ranging from 25 to $150 \mu \mathrm{M}$ in phosphate-buffered saline (PBS) (Figure $5 a-c)$. Next, to better simulate the SERS profile of UA in whole tears, we prepared various concentrations of UA between 25 and $150 \mu \mathrm{M}$ in an artificial tear buffer which consisted of various prominent tear proteins such as lysozyme, lactoferrin, albumin, and immunoglobulins, as well as electrolytes that maintain tear osmolarity such as $\mathrm{Na}^{+}, \mathrm{K}^{+}, \mathrm{Cl}^{-}$, and $\mathrm{HCO}_{3}{ }^{-}$(see the Methods Section in Supporting Information for additional details). As a practical demonstration of broadband SERS enhancement, the 640, 1134, and 1645 $\mathrm{cm}^{-1}$ peaks were tracked with excellent linearity being observed as shown in Figure 5d. These measurements from an artificial tear buffer were used as a characteristic curve to map measurements taken for whole tears. Finally, we analyzed the performance of the flex-MS toward the detection of UA levels in whole tears. Here, we tested pooled tears from eight different samples obtained from healthy subjects. As a comparison, the same measurement was made using a commercial colorimetric enzyme-based assay. The average concentration from the flex-MS for the 640, 1134, and 1645 $\mathrm{cm}^{-1}$ peaks $(66,67$, and $67 \mu \mathrm{M}$, respectively) was in good agreement with that from the enzyme-based assay $(76 \mu \mathrm{M})$ which demonstrates the potential of the flex-MS as an effective label-free SERS diagnostic platform (Figure 5e).

\section{CONCLUSIONS}

In summary, using a simple biomimetic fabrication process, we have developed a cost-effective, scalable, and flexible plasmonic metasurface-based platform for label-free SERS. The flex-MS consists of a dense ensemble of closely coupled Au nanodisks and $\mathrm{Au}$ nanoholes separated by sub-10 $\mathrm{nm} \mathrm{SiO}_{2}$ nanogaps in an MIM arrangement all on flexible PDMS thin films. The MIM provides a very tunable and large extinction cross section and a corresponding localized $|E|^{2}$ enhancement. When considering an ensemble of such MIM structures exhibiting controlled disorder, the resonance profile of the entire system becomes broadband thereby enabling the uniform enhancement of not just the excitation wavelength $\left(\lambda_{\mathrm{L}}\right)$, but also large Stokes-shifted Raman scattering wavelengths $\left(\lambda_{\mathrm{R}} \neq \lambda_{\mathrm{L}}\right)$. Finally, using flex-MS as a broadband diagnostic platform, we demonstrate the label-free detection of UA in both artificial tear buffer as well as whole human tear samples. Additionally, we compare the performance of the flex-MS with a commercial UA measurement assay and show that they are in good agreement. As a result, we envisage that through broadband SERS enhancement, the flex-MS can prove to be a reliable and scalable label-free diagnostic platform for a variety of SERSactive molecules.

\section{EXPERIMENTAL SECTION}

4.1. Biomimetic Flex-MS Fabrication. First, a $400 \mu \mathrm{m}$ thick PDMS layer (Sylgard 184 elastomer base mixed with curing agent in a ratio of 10:1, Dow Chemical Co., USA) was spin-coated on a $4 \mathrm{in}$. Si wafer and cured at $65^{\circ} \mathrm{C}$ for $12 \mathrm{~h}$. 
Next, poly(methyl methacrylate) (PMMA, $M_{w}=5090$, Polymer Standards Service GmbH, Germany) and polystyrene (PS, $M_{\mathrm{w}}=3250$, Polymer Standards Service GmbH, Germany) were co-dissolved in MEK (Sigma-Aldrich, USA) with mass ratios of $70 \%: 30 \%$. The solution concentrations were $20 \mathrm{mg}$ $\mathrm{mL}^{-1}$. After exposing the PDMS substrate to $\mathrm{O}_{2}$ plasma for 5 $\mathrm{min}$, the polymer blend solutions were spin-coated at a spinspeed of $3500 \mathrm{rpm}$ and acceleration of $2000 \mathrm{rpm} \mathrm{s}^{-1}$ for $30 \mathrm{~s}$. Relative humidity was maintained between 40 and 50\% during the spin-coating. As shown in Figure S1, the de-mixing of the blend components occurs during spin-coating because of the difference in relative solubility of PS and PMMA in MEK. First, water condensation begins at humidity levels above 35\% forming a water-rich layer at the air/solution interface because of the difference in the evaporation rate between water and MEK. Water starts to condense from the air into the solution because of the evaporation of MEK decreasing the temperature on the top, below the dew point. Because of the high water concentration, a 3D phase separation occurs between PS/MEK and PMMA/MEK/water. Upon drying, a purely lateral morphology was formed with ellipsoidal PS islands in a PMMA matrix. The samples were then rinsed in acetic acid for $60 \mathrm{~s}$ and dried in a stream of $\mathrm{N}_{2}$ to remove the PMMA matrix leaving behind the PS islands. The resulting PS nanopillar mask then served as a template for the E-beam evaporation of a $100 \pm 0.5$ nm-thick $\mathrm{SiO}_{2}$ layer (CHA MK40 E-Beam Evaporation, CHA Industries, USA). Next, a $91 \pm 1.1 \mathrm{~nm}-$ thick $\mathrm{Au}$ layer was deposited (CHA MK40 E-Beam Evaporation, $\mathrm{CHA}$ Industries, USA) over $\mathrm{SiO}_{2}$ to generate the MIM layer with $\sim 9 \pm 1.6 \mathrm{~nm}$ gap. Finally, the PDMS film was peeled off the Si wafer and served as a SERS substrate for biosensing experiments.

4.2. Periodic MIM Array Fabrication. PMMA950 A4 (MicroChem, USA) E-beam resist was spin-coated at 1500 rpm for $60 \mathrm{~s}$ onto an $\mathrm{Si}$ substrate cleaned with acetone and isopropyl alcohol prior to spin-coating. The sample was then exposed at a dosage of $800 \mu \mathrm{C} \mathrm{cm}^{-2}$ (Raith EBPG 5200, Raith Nanofabrication, Germany). Following exposure, development was carried out using a 1:1 ratio of IPA/MIBK for $1 \mathrm{~min}$. Next, $\mathrm{SiO}_{2}$ was directionally deposited via E-beam evaporation (CHA MK40 E-Beam Evaporation, CHA Industries, USA) after which lift-off was performed in Remover PG leaving behind $\mathrm{SiO}_{2}$ nanopillars. Finally, $\mathrm{Au}$ of an appropriate thickness was deposited (CHA MK40 E-Beam Evaporation, $\mathrm{CHA}$ Industries, USA) over $\mathrm{SiO}_{2}$ to generate the MIM layer.

4.3. High-Resolution Imaging and Statistical Analyses of the Flex-MS. SEM imaging was performed on the flex-MS using a Nova 200 Novalab Dualbeam microscope (FEI, USA) at $10 \mathrm{kV}$. ImageJ (National Institutes of Health, USA), a Java-based public-domain image processing tool was used to obtain the diameter distribution of the nanostructures. Every pixel in the acquired SEM images were converted to black or white based on a thresholding condition that was obtained by calculating the mean intensity value of all pixels of the image. Following this, the diameter distribution of the MIM scatterers was determined. Finally, the FFT analysis to determine short-range periodicity was performed using MATLAB (MathWorks, USA).

4.4. Optical Simulations of the Flex-MS. Optical simulations of a single MIM nanostructure as well as the quasi-ordered/periodic ensemble as a whole were performed using 3D finite-difference time-domain software (Lumerical Solutions, Canada). A combination of periodic and perfect matching layer boundary conditions along with a plane wave source was used. The absorption cross section was obtained in the total field region inside the source while the scattering cross section was obtained in the scattered field region outside the source. The influence of the nanogap was studied as shown in Figure S6. The $\left|E / E_{0}\right|^{2}$ enhancement was obtained by placing a frequency-domain field monitor at the MIM junction spanning the entire MIM nanostructure or the ensemble, respectively. The field maps as well as the effective $\left|E / E_{0}\right|^{2}$ enhancement (averaged over 4000 individual hotspots or mesh cells) in the case of the ensemble were calculated using MATLAB (MathWorks, USA).

4.5. Spectroscopic Analyses. An optical microscope operating in dark-field (DF) mode was used for the microspectroscopic investigation of the fabricated flex-MS and periodic MIM array samples. A halogen lamp was used as a light source using a $50 \times$ objective. The scattered light was collected in a confocal configuration and analyzed using a spectrometer (AvaSpec-ULS2048x64-USB2). A $400 \mu \mathrm{m}$ core optical fiber was used to obtain a spatial resolution of $20 \mu \mathrm{m}$ to characterize the scattering of the samples.

4.6. SERS Measurements. All SERS measurements were performed by incubating a $30 \mu \mathrm{L}$ droplet of the reagent on the flex-MS for $30 \mathrm{~min}$. Following this, the drop was dislodged from the sample surface using a stream of $\mathrm{N}_{2}$. The sample was then measured dry using a Raman microscope (inVia, Renishaw, United Kingdom) with a $50 \times$ objective lens. A $785 \mathrm{~nm}$ laser operated at $0.32,0.62$, or $1.12 \mathrm{~mW}$ for a duration of $60 \mathrm{~s}$ was used to take the measurements.

4.7. Artificial Tear Buffer Preparation. Artificial tear buffer was prepared using previously reported methods. ${ }^{10,42}$ Briefly, electrolytes $\mathrm{K}^{+}, \mathrm{HCO}_{3}{ }^{-}, \mathrm{Na}^{+}$, and $\mathrm{Cl}^{-}$were added to DI water at concentrations of $24,24,130$, and $130 \mathrm{mM}$, respectively. Following this, representative tear proteins such as lysozyme, lactoferrin, albumin, and IgG were added at concentrations of $2 \mathrm{mg} \mathrm{mL}^{-1}, 2 \mathrm{mg} \mathrm{mL}^{-1}, 0.02 \mathrm{mg} \mathrm{mL}^{-1}$, and $3 \mu \mathrm{g} \mathrm{mL}^{-1}$ respectively.

4.8. Whole Tear Assay. Eight individual tear samples were collected from healthy subjects and pooled for the experiment. For a comparative study, a commercial enzyme-based colorimetric UA detection kit (Sigma-Aldrich, USA) was used. The assay was performed following the manufacturer's protocol.

\section{ASSOCIATED CONTENT}

\section{Supporting Information}

The Supporting Information is available free of charge at https://pubs.acs.org/doi/10.1021/acsomega.0c00677.

Schematic of the different stages of the 3D phase separation process, characterization of the flex-MS FDTD model, extinction cross section as a function of the nanogap size, comparison of $\left|E / E_{\mathrm{o}}\right|^{2}$ enhancement from a single MIM structure and a periodic array, simulated flex-MS FDTD model, comparison of an MIM structure with an open and covered nanogap, and suppression of background signal by the flex-MS (PDF)

\section{AUTHOR INFORMATION}

\section{Corresponding Author}

Hyuck Choo - Department of Electrical Engineering, California Institute of Technology, Pasadena, California 91125, United States; Samsung Advanced Institute of Technology, Samsung 
Electronics, Suwon, Gyeonggi-do 16678, South Korea; Email: hyuck.choo@samsung.com, hchoo@caltech.edu

\section{Authors}

Vinayak Narasimhan - Department of Medical Engineering, California Institute of Technology, Pasadena, California 91125, United States; 10 orcid.org/0000-0003-4165-402X

Radwanul Hasan Siddique - Department of Medical Engineering, California Institute of Technology, Pasadena, California 91125, United States; Image Sensor Lab, Samsung Semiconductor, Inc., Pasadena, California 91101, United States; O orcid.org/0000-0001-7494-5857

Haeri Park - Department of Medical Engineering, California Institute of Technology, Pasadena, California 91125, United States

Complete contact information is available at: https://pubs.acs.org/10.1021/acsomega.0c00677

\section{Author Contributions}

V.N., R.H.S., and H.C. conceived the study. V.N., R.H.S., and H.P. performed the necessary simulations and experiments under the supervision of H.C. The manuscript was written through contributions of all the authors.

\section{Funding}

SAMSUNG Global Research Outreach (GRO) program.

\section{Notes}

The authors declare no competing financial interest.

\section{ACKNOWLEDGMENTS}

The authors acknowledge the financial support provided by the SAMSUNG Global Research Outreach (GRO) program. The authors are also thankful for the support and resources provided by the Kavli Nanoscience Institute at Caltech.

\section{REFERENCES}

(1) Nie, S.; Emory, S. R. Probing Single Molecules and Single Nanoparticles by Surface-Enhanced Raman Scattering. Science 1997, 275, 1102-1106.

(2) Sharma, B.; Frontiera, R. R.; Henry, A.-I.; Ringe, E.; Van Duyne, R. P. SERS: Materials, Applications, and the Future. Mater. Today 2012, 15, 16-25.

(3) Langer, J.; Jimenez de Aberasturi, D.; Aizpurua, J.; AlvarezPuebla, R. A.; Auguié, B.; Baumberg, J. J.; Bazan, G. C.; Bell, S. E. J.; Boisen, A.; Brolo, A. G.; Choo, J.; Cialla-May, D.; Deckert, V.; Fabris, L.; Faulds, K.; García de Abajo, F. J.; Goodacre, R.; Graham, D.; Haes, A. J.; Haynes, C. L.; Huck, C.; Itoh, T.; Käll, M.; Kneipp, J.; Kotov, N. A.; Kuang, H.; Le Ru, E. C.; Lee, H. K.; Li, J.-F.; Ling, X. Y.; Maier, S. A.; Mayerhöfer, T.; Moskovits, M.; Murakoshi, K.; Nam, J.-M.; Nie, S.; Ozaki, Y.; Pastoriza-Santos, I.; Perez-Juste, J.; Popp, J.; Pucci, A.; Reich, S.; Ren, B.; Schatz, G. C.; Shegai, T.; Schlücker, S.; Tay, L.-L.; Thomas, K. G.; Tian, Z.-Q.; Van Duyne, R. P.; Vo-Dinh, T.; Wang, Y.; Willets, K. A.; Xu, C.; Xu, H.; Xu, Y.; Yamamoto, Y. S.; Zhao, B.; Liz-Marzán, L. M. Present and Future of Surface-Enhanced Raman Scattering. ACS Nano 2020, 14, 28.

(4) Xu, K.; Wang, Z.; Tan, C. F.; Kang, N.; Chen, L.; Ren, L.; Thian, E. S.; Ho, G. W.; Ji, R.; Hong, M. Uniaxially Stretched Flexible Surface Plasmon Resonance Film for Versatile Surface Enhanced Raman Scattering Diagnostics. ACS Appl. Mater. Interfaces 2017, 9, 26341-26349.

(5) Cui, H.; Li, S.; Deng, S.; Chen, H.; Wang, C. Flexible, Transparent, and Free-Standing Silicon Nanowire SERS Platform for in Situ Food Inspection. ACS Sens. 2017, 2, 386-393.

(6) Xu, K.; Zhou, R.; Takei, K.; Hong, M. Toward Flexible SurfaceEnhanced Raman Scattering (SERS) Sensors for Point-of-Care Diagnostics. Adv. Sci. 2019, 6, 1900925.
(7) Kang, H.; Heo, C.-J.; Jeon, H. C.; Lee, S. Y.; Yang, S.-M. Durable Plasmonic Cap Arrays on Flexible Substrate with Real-Time Optical Tunability for High-Fidelity SERS Devices. ACS Appl. Mater. Interfaces 2013, 5, 4569-4574.

(8) Jeong, J. W.; Yang, S. R.; Hur, Y. H.; Kim, S. W.; Baek, K. M.; Yim, S.; Jang, H.-I.; Park, J. H.; Lee, S. Y.; Park, C.-O.; Jung, Y. S. High-Resolution Nanotransfer Printing Applicable to Diverse Surfaces via Interface-Targeted Adhesion Switching. Nat. Commun. 2014, 5, 5387.

(9) Tian, L.; Jiang, Q.; Liu, K.-K.; Luan, J.; Naik, R. R.; Singamaneni, S. Bacterial Nanocellulose-Based Flexible Surface Enhanced Raman Scattering Substrate. Adv. Mater. Interfaces 2016, 3, 1600214.

(10) Park, M.; Jung, H.; Jeong, Y.; Jeong, K.-H. Plasmonic Schirmer Strip for Human Tear-Based Gouty Arthritis Diagnosis Using SurfaceEnhanced Raman Scattering. ACS Nano 2017, 11, 438-443.

(11) Messina, G. C.; Malerba, M.; Zilio, P.; Miele, E.; Dipalo, M.; Ferrara, L.; De Angelis, F. Hollow Plasmonic Antennas for Broadband SERS Spectroscopy. Beilstein J. Nanotechnol. 2015, 6, 492-498.

(12) Li, M.; Xu, J.; Romero-Gonzalez, M.; Banwart, S. A.; Huang, W. E. Single Cell Raman Spectroscopy for Cell Sorting and Imaging. Curr. Opin. Biotechnol. 2012, 23, 56-63.

(13) De Angelis, F.; Malerba, M.; Patrini, M.; Miele, E.; Das, G.; Toma, A.; Zaccaria, R. P.; Di Fabrizio, E. 3D Hollow Nanostructures as Building Blocks for Multifunctional Plasmonics. Nano Lett. 2013, 13, 3553-3558.

(14) Lin, X.-M.; Cui, Y.; Xu, Y.-H.; Ren, B.; Tian, Z.-Q. SurfaceEnhanced Raman Spectroscopy: Substrate-Related Issues. Anal. Bioanal. Chem. 2009, 394, 1729-1745.

(15) Wu, L.; Wang, W.; Zhang, W.; Su, H.; Liu, Q.; Gu, J.; Deng, T.; Zhang, D. Highly Sensitive, Reproducible and Uniform SERS Substrates with a High Density of Three-Dimensionally Distributed Hotspots: Gyroid-Structured Au Periodic Metallic Materials. NPG Asia Mater. 2018, 10, No. e462.

(16) Johansen, V. E.; Onelli, O. D.; Steiner, L. M.; Vignolini, S. Photonics in Nature: From Order to Disorder. In Functional Surfaces in Biology III: Diversity of the Physical Phenomena; Gorb, S. N., Gorb, E. V., Eds.; Biologically-Inspired Systems; Springer International Publishing: Cham, 2017; pp 53-89.

(17) Kolle, M.; Lee, S. Progress and Opportunities in Soft Photonics and Biologically Inspired Optics. Adv. Mater. 2018, 30, 1702669.

(18) Wiersma, D. S. Disordered Photonics. Nat. Photonics 2013, 7, $188-196$.

(19) Florescu, M.; Torquato, S.; Steinhardt, P. J. Designer Disordered Materials with Large, Complete Photonic Band Gaps. Proc. Natl. Acad. Sci. U.S.A. 2009, 106, 20658-20663.

(20) Narasimhan, V.; Siddique, R. H.; Lee, J. O.; Kumar, S.; Ndjamen, B.; Du, J.; Hong, N.; Sretavan, D.; Choo, H. Multifunctional Biophotonic Nanostructures Inspired by the Longtail Glasswing Butterfly for Medical Devices. Nat. Nanotechnol. 2018, 13, 512-519.

(21) Siddique, R. H.; Donie, Y. J.; Gomard, G.; Yalamanchili, S.; Merdzhanova, T.; Lemmer, U.; Hölscher, H. Bioinspired PhaseSeparated Disordered Nanostructures for Thin Photovoltaic Absorbers. Sci. Adv. 2017, 3, No. e1700232.

(22) Saranathan, V.; Seago, A. E.; Sandy, A.; Narayanan, S.; Mochrie, S. G. J.; Dufresne, E. R.; Cao, H.; Osuji, C. O.; Prum, R. O. Structural Diversity of Arthropod Biophotonic Nanostructures Spans Amphiphilic Phase-Space. Nano Lett. 2015, 15, 3735-3742.

(23) Siddique, R. H.; Kumar, S.; Narasimhan, V.; Kwon, H.; Choo, H. Aluminum Metasurface with Hybrid Multipolar Plasmons for 1000-Fold Broadband Visible Fluorescence Enhancement and Multiplexed Biosensing. ACS Nano 2019, 13, 13775.

(24) Narasimhan, V.; Siddique, R. H.; Hoffmann, M.; Kumar, S.; Choo, H. Enhanced Broadband Fluorescence Detection of Nucleic Acids Using Multipolar Gap-Plasmons on Biomimetic Au Metasurfaces. Nanoscale 2019, 11, 13750-13757.

(25) Yang, Y.; Hu, Z.; Wang, Y.; Wang, B.; Zhan, Q.; Zhang, Y.; Ao, $\mathrm{X}$. Broadband SERS Substrates by Oblique Angle Deposition Method. Opt. Mater. Express 2016, 6, 2644-2654. 
(26) Wang, D.; Zhu, W.; Best, M. D.; Camden, J. P.; Crozier, K. B. Wafer-Scale Metasurface for Total Power Absorption, Local Field Enhancement and Single Molecule Raman Spectroscopy. Sci. Rep.

2013, 3, 1-6.

(27) Kravets, V. G.; Kabashin, A. V.; Barnes, W. L.; Grigorenko, A. N. Plasmonic Surface Lattice Resonances: A Review of Properties and Applications. Chem. Rev. 2018, 118, 5912-5951.

(28) Auguié, B.; Barnes, W. L. Diffractive Coupling in Gold Nanoparticle Arrays and the Effect of Disorder. Opt. Lett. 2009, 34, 401-403.

(29) Bertin, H.; Brûlé, Y.; Magno, G.; Lopez, T.; Gogol, P.; Pradere, L.; Gralak, B.; Barat, D.; Demésy, G.; Dagens, B. Correlated Disordered Plasmonic Nanostructures Arrays for Augmented Reality. ACS Photonics 2018, 5, 2661-2668.

(30) Ding, S.-Y.; You, E.-M.; Tian, Z.-Q.; Moskovits, M. Electromagnetic Theories of Surface-Enhanced Raman Spectroscopy. Chem. Soc. Rev. 2017, 46, 4042-4076.

(31) Le Ru, E. C.; Etchegoin, P. G. SERS Enhancement Factors and Related Topics; Le Ru, E. C., Etchegoin, P. G., Eds.; Elsevier: Amsterdam, 2009.

(32) Stiles, P. L.; Dieringer, J. A.; Shah, N. C.; Van Duyne, R. P. Surface-Enhanced Raman Spectroscopy. Annu. Rev. Anal. Chem. 2008, $1,601-626$.

(33) McFarland, A. D.; Young, M. A.; Dieringer, J. A.; Van Duyne, R. P. Wavelength-Scanned Surface-Enhanced Raman Excitation Spectroscopy. J. Phys. Chem. B 2005, 109, 11279-11285.

(34) Schlücker, S. Surface-Enhanced Raman Spectroscopy: Concepts and Chemical Applications. Angew. Chem., Int. Ed. 2014, 53, 4756-4795.

(35) Westley, C.; Xu, Y.; Thilaganathan, B.; Carnell, A. J.; Turner, N. J.; Goodacre, R. Absolute Quantification of Uric Acid in Human Urine Using Surface Enhanced Raman Scattering with the Standard Addition Method. Anal. Chem. 2017, 89, 2472-2477.

(36) Reginato, A. M.; Mount, D. B.; Yang, I.; Choi, H. K. The Genetics of Hyperuricaemia and Gout. Nat. Rev. Rheumatol. 2012, 8, 610-621.

(37) Bhole, V.; Choi, J. W. J.; Woo Kim, S.; de Vera, M.; Choi, H. Serum Uric Acid Levels and the Risk of Type 2 Diabetes: A Prospective Study. Am. J. Med. 2010, 123, 957-961.

(38) Wen, M.; Zhou, B.; Chen, Y.-H.; Ma, Z.-L.; Gou, Y.; Zhang, C.L.; Yu, W.-F.; Jiao, L. Serum Uric Acid Levels in Patients with Parkinson's Disease: A Meta-Analysis. PLoS One 2017, 12, No. e0173731.

(39) Kohagura, K.; Kochi, M.; Miyagi, T.; Kinjyo, T.; Maehara, Y.; Nagahama, K.; Sakima, A.; Iseki, K.; Ohya, Y. An Association between Uric Acid Levels and Renal Arteriolopathy in Chronic Kidney Disease: A Biopsy-Based Study. Hypertens. Res. 2013, 36, 43-49.

(40) Feig, D. I.; Kang, D.-H.; Johnson, R. J. Uric Acid and Cardiovascular Risk. N. Engl. J. Med. 2008, 359, 1811-1821.

(41) Hagan, S.; Martin, E.; Enríquez-de-Salamanca, A. Tear Fluid Biomarkers in Ocular and Systemic Disease: Potential Use for Predictive, Preventive and Personalised Medicine. EPMA J. 2016, 7, 15.

(42) Culver, H. R.; Wechsler, M. E.; Peppas, N. A. Label-Free Detection of Tear Biomarkers Using Hydrogel-Coated Gold Nanoshells in a Localized Surface Plasmon Resonance-Based Biosensor. ACS Nano 2018, 12, 9342-9354. 